\title{
Concept, Implementation, and Control of Wide Bandwidth MEMS Gyroscopes
}

\author{
Cenk Acar, Sebnem Eler, and Andrei M. Shkel \\ Microsystems Laboratory \\ Mechanical and Aerospace Engineering Department \\ University of California at Irvine \\ Irvine, California 92697
}

\begin{abstract}
This paper reports a novel concept for designing widebandwidth micromachined gyroscopes with improved robustness. The approach suggests the use of two independently oscillating interconnected proof masses to form a 4-DOF dynamical system, and thus increasing the design parameter space of the inertial system. The concept is implemented using MEMS technology. Computer modeling of the proposed design indicates over 15 times increase in the bandwidth of the system as compared to the conventional gyroscopes. In addition, significantly reduced sensitivity of the gyroscope to structural and thermal parameter fluctuations and damping is demonstrated. By utilizing the disturbance-rejection capability of the inertial system, improved robustness is achieved without sophistication in control electronics. All these advantages of the proposed design might relax strict fabrication tolerances and packaging requirements, reducing production cost of micromachined gyroscopes.
\end{abstract}

\section{Introduction}

With the advances in micromachining technologies, low cost inertial micro-sensors on-a-chip are beginning to enter the market. Derived from the conventional Integrated Circuit (IC) fabrication technologies, micromachining processes allow mass-production of microstructures with moving parts on a chip controlled by electronics integrated on the same chip. Optimistic projections predict that in a near future, expensive and bulky conventional inertial sensors will be replaced by their low-cost and micro-sized counterparts without any compromise in performance. Micromachined gyroscopes could potentially provide high accuracy rotation measurements leading to a wide range of applications including navigation and guidance systems, automotive safety systems, and consumer electronics. Gyroscopes are probably the most challenging type of transducers ever attempted to be designed using MEMS technology.

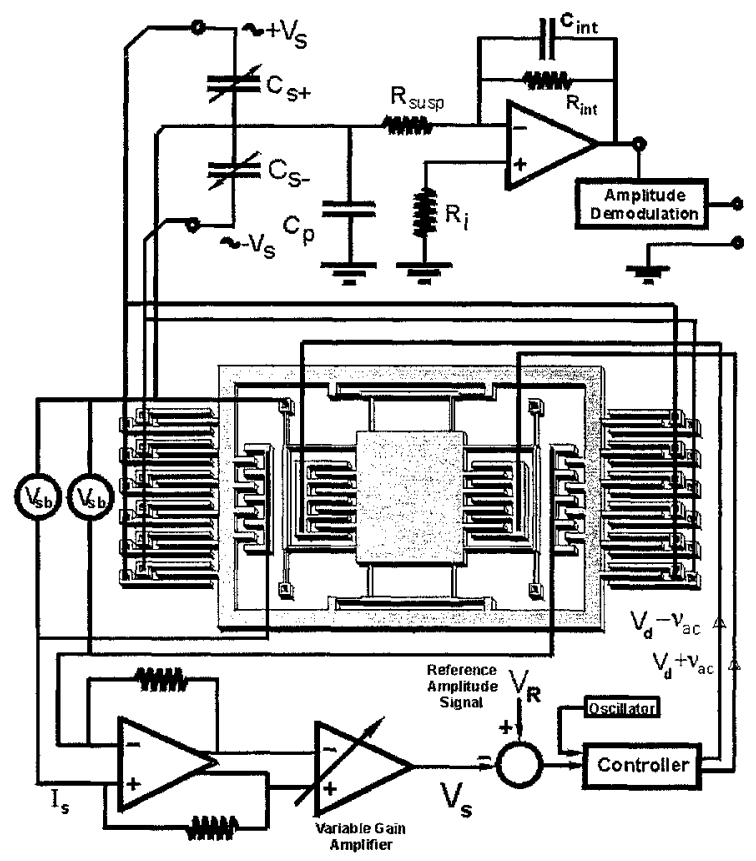

Figure 1: Illustration of the proposed micromachined gyroscope with control and sense electronics.

Truly low-cost and high-performance devices are not on the market yet, and the current state of the art micromachined gyroscopes require an order of magnitude improvement in performance, stability, and robustness.

All existing micromachined rate gyroscopes operate on the vibratory principle of a single proof mass suspended by flexures anchored to the substrate. The flexures serve as the flexible suspension between the proof mass and the substrate, making the mass free to oscillate in two orthogonal directions (Fig. 2a): the drive (x-axis) and the sense (y-axis) [1]. The proof mass is driven into resonance in the $\mathrm{x}$-direction, and in the presence of an angular rotation, the Coriolis force proportional to the input angular rate is induced in the y-direction. 
To achieve the maximum possible gain, the conventional gyroscopes are designed to operate at the peak of the response curve. This is typically achieved by matching drive and sense resonant frequencies (Fig. $2 c)$. In result, the system is very sensitive to variations in system parameters causing a shift in the resonant frequency. Under high quality factor conditions the gain is high, however the bandwidth is extremely narrow. For example, a 1\% fluctuation in frequency matching between drive and sense modes will produce an error of $20 \%$ in the output signal gain [3]. In addition, the gain is affected significantly by fluctuations in damping (Fig. 2c). Generally, very sophisticated control electronics is used to provide operation in the region of the resonance peak [4].

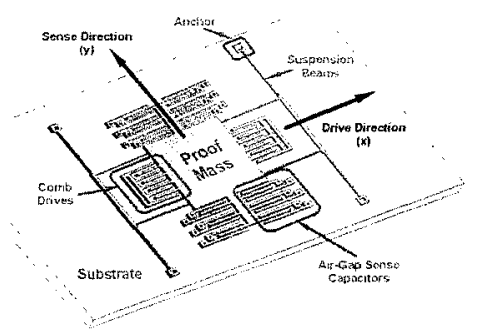

(a)

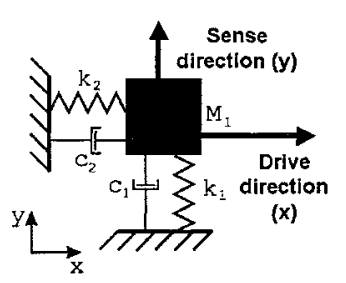

(b)

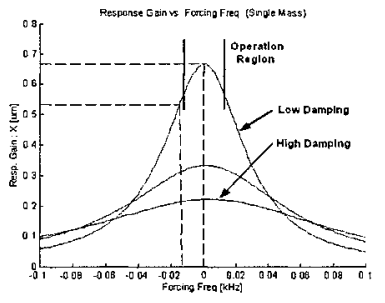

(c)
Figure 2: (a) Conceptual design of a conventional micromachined rate gyroscope. (b) The dynamical system has 2 degrees-of-freedom. (c) The response is sensitive to fluctuations in damping and drive frequency variations.

To eliminate the limitations of the existing micromachined gyroscopes, a design approach that suggests the use of two independently vibrating proof masses to form a 4-DOF dynamical system is presented in this paper.

\section{Design Approach and Principle of Operation}

In contrast to the conventional micromachined gyroscopes, the proposed design approach utilizes two vibrating proof masses suspended above the substrate, which form a 4 degrees-of-freedom (DOF) dynamic system (Fig. 4). The gyroscope has two orthogonal principle axes of oscillation: the drive direction ( $x$ axis in Fig- ure 3) and the sense direction (y axis in Figure 3). The suspension system renders both of the proof masses free to oscillate in the drive and sense directions.

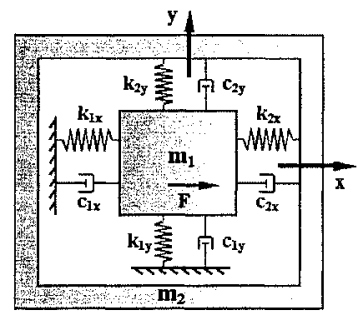

Figure 3: Lumped 4-DOF mass-spring-damper model of the dual-mass gyroscope.

The first mass $\left(m_{1}\right.$ in Figure 3$)$ is electrostatically forced to oscillate in the drive direction, and this forced oscillation is amplified by the second mass $\left(m_{2}\right.$ in Figure 3). The response of the second mass in the orthogonal sense direction is monitored. The resulting 4DOF dynamic system has a more favorable frequency response, and can operate in a wider frequency band with insignificant change in the gain. Furthermore, the increased number of degrees-of-freedom results in an increased design parameter space, which allows the dynamic response of the gyroscope to be shaped as needed with much less compromise in performance. An implementation of the conceptual design, Fig. 3, is illustrated in Fig. 4 (see details in [5]).

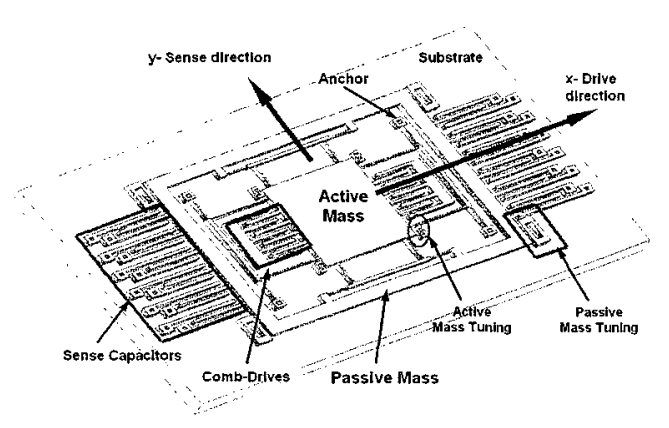

Figure 4: Schematic illustration of a MEMS implementation of the dual-mass z-axis gyroscope.

With appropriate selection of dynamical system parameters including the masses and the spring rates, one can obtain the frequency response illustrated in Fig.5. There exists three regions of interest on this response curve: two resonant peaks, regions 1 and 3; and a flat region between the peaks, region 2 . According to the proposed design approach, the nominal operation of the gyroscope is in the flat region, where the signal gain is relatively high, and the sensitivity of the gain to driving frequency variations is low. Because of the widened bandwidth, a $1 \%$ variation in natural frequencies of the 
system results in only $0.8 \%$ error in the output signal, whereas the same fluctuation will produce an error of $20 \%$ in the conventional micromachined gyroscopes [3].

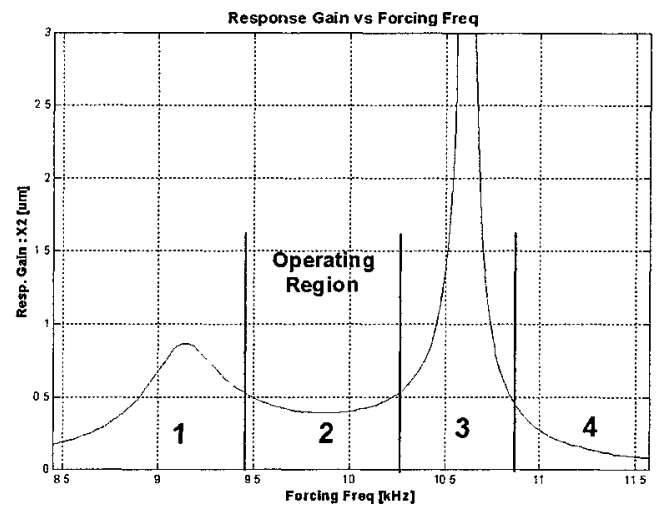

Figure 5: Response of the dual-mass gyroscope in the flat operation region is insensitive to resonant frequency fluctuations and has over 15 times wider bandwidth than in conventional gyroscopes.

\section{Dynamics of the Gyroscope}

The dynamics of the gyroscope should be considered in the non-inertial frame. When the active and passive masses are observed in the rotating gyroscope frame, additional inertial forces appear acting on both masses. The equations of motion for the two-mass system can be expressed as:

$$
\begin{aligned}
& m_{1} \overrightarrow{a_{1}}=\overrightarrow{F_{2-1}}+\overrightarrow{F_{s-1}}-2 m_{1} \vec{\Omega} \times \overrightarrow{v_{1}}-m_{1} \vec{\Omega} \times\left(\vec{\Omega} \times \overrightarrow{r_{1}}\right) \\
& m_{2} \overrightarrow{a_{2}}=\overrightarrow{F_{1-2}}+\overrightarrow{F_{s-2}}-2 m_{2} \vec{\Omega} \times \overrightarrow{v_{2}}-m_{2} \vec{\Omega} \times\left(\vec{\Omega} \times \overrightarrow{r_{2}}\right)
\end{aligned}
$$

where $\overrightarrow{r_{1}}$ and $\overrightarrow{r_{2}}$ are the position vectors, $\overrightarrow{v_{1}}$ and $\overrightarrow{v_{2}}$ are the velocity vectors of the masses defined in the gyroscope frame, $\overrightarrow{F_{2-1}}$ and $\overrightarrow{F_{1-2}}$ are the coupling spring and damping forces between the masses proportional to the relative position and velocity. $\overrightarrow{F_{s-1}}$, and $\overrightarrow{F_{s-2}}$ are the active mass - substrate, and passive mass - substrate forces, respectively. The term $2 m_{2} \vec{\Omega} \times \overrightarrow{v_{2}}$, the Coriolis force, is of special interest since the operation of the gyroscope depends on excitation of system in the sense direction by this force. Since both masses are subject to an angular rate, $\Omega_{z}$, about the axis normal to the plane of operation (z-axis), the equations of motion along the $\mathrm{x}$-axis and $\mathrm{y}$-axis become:

$m_{1} \ddot{x_{1}}+c_{1 x} \dot{x_{1}}+k_{1 x} x_{1}=k_{2 x}\left(x_{2}-x_{1}\right)+c_{2 x}\left(\dot{x_{2}}-\dot{x_{1}}\right)+$ $m_{1} \Omega^{2} x_{1}-2 m_{1} \Omega \dot{y_{1}}+m_{1} \dot{\Omega} y_{1}+F_{d}(t)$

$m_{2} \ddot{x_{2}}+c_{2 x}\left(\dot{x_{2}}-\dot{x_{1}}\right)+k_{2 x}\left(x_{2}-x_{1}\right)=$

$m_{2} \Omega^{2} x_{2}-2 m_{2} \Omega \dot{y}_{2}+m_{2} \dot{\Omega} y_{2}$

$m_{1} \ddot{y_{1}}+c_{1 y} \dot{y}_{1}+k_{1 y} y_{1}=$

$k_{2 y}\left(y_{2}-y_{1}\right)+c_{2 y}\left(\dot{y_{2}}-\dot{y_{1}}\right)+m_{1} \Omega^{2} y_{1}+2 m_{1} \Omega \dot{x_{1}}+m_{1} \dot{\Omega} x_{1}$

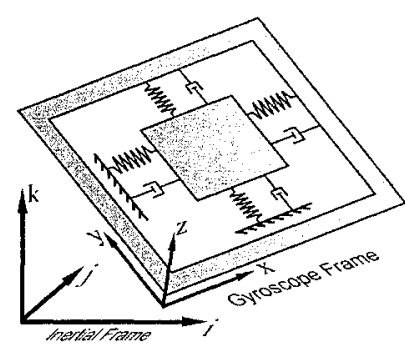

Figure 6: Representation of the gyroscope dynamics relative to the rotating gyroscope frame.

$m_{2} \ddot{y_{2}}+c_{2 y}\left(\dot{y_{2}}-\dot{y_{1}}\right)+k_{2 y}\left(y_{2}-y_{1}\right)=$ $m_{2} \Omega^{2} y_{2}+2 m_{2} \Omega \dot{x_{2}}+m_{2} \dot{\Omega} x_{2}$.

where $F_{d}(t)$ is the driving electrostatic force applied to the active mass, and $\Omega$ is the angular velocity applied to the gyroscope about the z-axis.

The overall dynamic model can be reduced having the active mass driven into forced oscillation in drive direction by $F_{d}(t)$ with a constant amplitude $x_{o}$ and a frequency $\omega_{d}$. Assuming the oscillation of the first mass in the drive direction is set by the control system to be

$$
x_{1}=x_{o} \cos \left(\omega_{d} t\right),
$$

the system reduces to three degrees-of-freedom. The equations of motion of the reduced system become [3]:

$\ddot{y_{1}}+2 \omega_{n} \xi \dot{y_{1}}+2 \mu \omega_{n} \xi\left(\dot{y_{1}}-\dot{y_{2}}\right)+\left(\omega_{n}-\Omega\right) y_{1}+$

$\omega_{n}^{2} \sigma_{1}\left(y_{1}-y_{2}\right)=-2 \Omega \omega_{d} x_{o} \sin \omega_{d} t+\dot{\Omega} x_{o} \cos \omega_{d} t$

$\beta\left(\ddot{y_{2}}-\Omega^{2} y_{2}\right)+2 \mu \omega_{n} \xi\left(\dot{y_{2}}-\dot{y_{1}}\right)-2 \beta \Omega \dot{x_{2}}-\beta \dot{\omega}_{z} x_{2}+$

$\omega_{n}^{2} \sigma_{1}\left(y_{2}-y_{1}\right)=0$

$\beta\left(\ddot{x_{2}}-\Omega^{2} x_{2}\right)+2 \beta \Omega \dot{y}_{2}+\beta \dot{\Omega} y_{2}+\omega_{n}^{2} \sigma_{2} x_{2}=w_{n}^{2} \sigma_{2} x_{o} \cos \omega_{d} t$

where $\beta=m_{2} / m_{1}, \sigma_{1}=k_{2 y} / k_{1 y}, \sigma_{2}=k_{2 x} / k_{1 x}$, $\mu=c_{2} / c_{1}, \xi=c_{1} /\left(2 m_{1} w_{n}\right)$, and $w_{n}$ is the natural frequency in the sense direction. Proper selection of system parameters will result in the frequency response illustrated in Fig. 5.

\section{Control System Issues}

Since the Coriolis response of the second mass in the sense direction is directly correlated with the drive oscillation amplitude, it is crucial that the second mass is driven into forced oscillation in drive direction with a known constant amplitude. Thus, the primary task of the control electronics is to assure constant amplitude oscillation for the second mass with the required driving frequency.

Moreover, an ideal gyroscope should be sensitive to only the input angular rate. However, in practice, micromachined gyroscopes are sensitive not only to the 
measured angular rate, but also to fabrication imperfections and undesirable excitations. Thus, the other major task of the control system is to compensate for these imperfections.

In the conventional gyroscope design approaches, sophisticated control strategies are required to set the oscillation amplitude to a constant desired value, and at the same time to adjust the driving frequency providing the maximum gain. However, since the proposed design is less sensitive to driving frequency and system parameter variations, less demanding control strategies are demonstrated to provide proper operation.

\subsection{Control of Oscillations in Drive Mode}

The Coriolis force which excites the second mass in the sense direction is described by $F_{\text {Coriolis }}=2 m_{2} \Omega \dot{x_{2}}$. Since the Coriolis force is proportional to the linear velocity of the second mass, it is also proportional to the oscillation amplitude. To assure an accurate angular rate measurement, the drive mode oscillation amplitude of the second mass should be kept constant by the drive electronics. This can be achieved either with an open-loop control or with a closed-loop control.

4.1.1 Open-Loop Control: The simplest approach for controlling the drive-mode oscillations is the open-loop control. The gyroscope is simply a 2-DOF system in the drive mode. The drive force is applied to the first mass by the comb drive structures (Figure 7b). Approximating the gyroscope by a lumped mass-spring-damper model (Figure 7a), the equations of motion in the drive direction can be expressed as

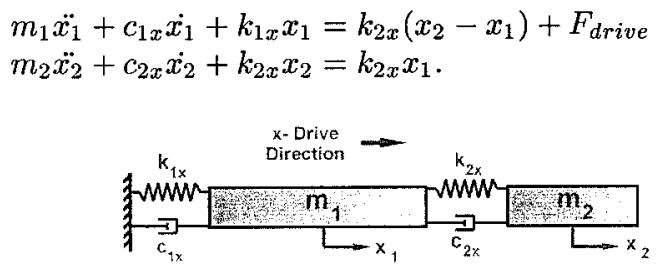

(a)

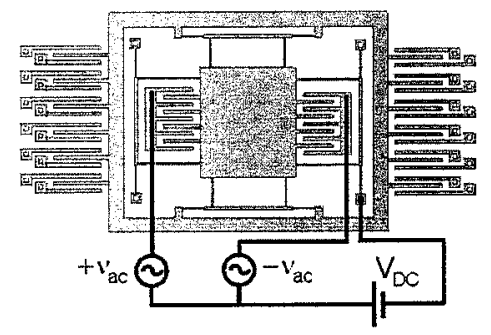

(b)

Figure 7: (a) Drive mode lumped mass-spring-damper model of a dual-mass gyroscope. (b) Open-loop driving scheme of the dual-mass gyroscope.

Employing balanced comb-drive strategy (Figure 7b) to drive the system with a frequency of $\omega_{\text {drive }}$, a net force linear to the amplitude of the alternating voltage $\nu_{A C}=\left|\nu_{A C}\right| \sin \left(\omega_{\text {drive }} t\right)$ and the DC bias voltage $V_{D C}$ can be achieved. When a voltage of $V_{1}=V_{D C}+\nu_{A C}$ is applied to one set of comb drives, and a voltage of $V_{2}=V_{D C}-\nu_{A C}$ is applied to the other set, the net electrostatic force can be approximated as:

$$
F=4 \frac{\varepsilon_{0} z_{0} N}{y_{0}} V_{D C} \nu_{A C}
$$

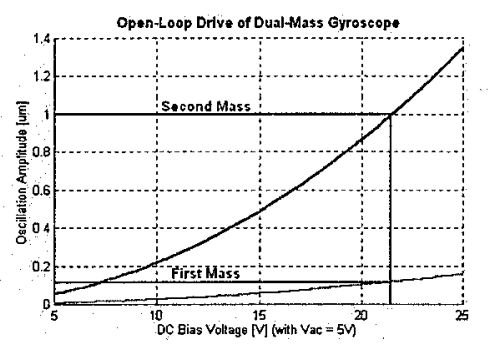

Figure 8: Oscillation amplitudes of the active and passive masses depending on applied voltages.

where $N$ is the number of comb-drive fingers, $z_{0}$ is the finger thickness, and $y_{0}$ is the finger separation. This driving scheme can be realized using the setup illustrated in Figure 9. Results of the computer modeling of the 2-DOF dynamic system with this force profile indicate that $1 \mu \mathrm{m}$ oscillation amplitude of the second mass is achieved with $5 \mathrm{~V} \mathrm{AC}$ and about $22 \mathrm{~V} \mathrm{DC}$ bias voltage (Figure 8).

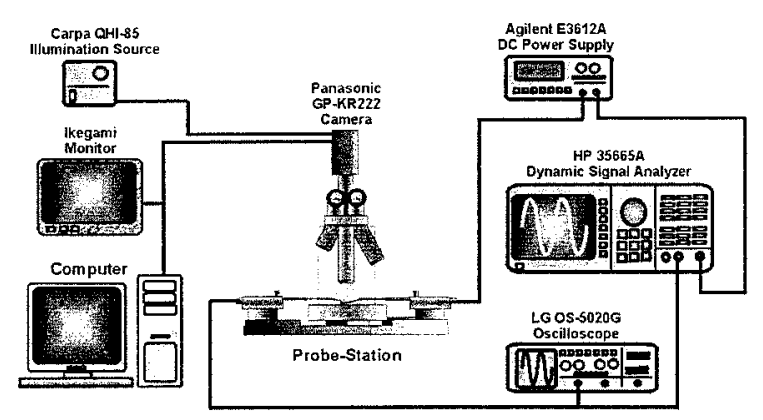

Figure 9: Experimental setup for open-loop driving.

4.1.2 Closed-Loop Control: Since it is vital to have a known drive-direction oscillation amplitude of the second mass for accurate rate measurement, the error in the oscillation amplitude should be minimized. When the gyroscope is driven using an open-loop control, any change in system parameters will cause errors in the oscillation amplitude, leading to erroneous rate measurement. Thus, it is necessary to have feedback control to achieve the desired oscillation amplitude.

To provide the feedback signal for the control system, comb structures have to be built on the second mass 
(Figure 10). If a trans-resistance amplifier is incorporated into the control loop, the displacement current generated by the oscillating second mass will be converted to an output voltage. This output voltage $V_{s}$ is then compared to a reference voltage $V_{R}$, which determines the desired oscillation amplitude.

The current due to capacitance change in the comb drives with the motion of the second mass can be expressed in terms of linear velocity of the second mass $\nu_{2}$, capacitance change due to deflection $\frac{\partial C}{\partial x}$, deflection sense bias voltage $V_{s b}$, and amplitude of oscillation $X_{2}$ as:

$$
I_{s}=V_{s b} \frac{\partial C}{\partial x} \nu_{2}(t)=V_{s b} \frac{\partial C}{\partial x} X_{2} \omega_{\text {drive }} \sin \left(\omega_{\text {drive }} t\right)
$$

With this current input, the output voltage from the trans-resistance amplifier, which will be compared to the reference input to get the error signal, is:

$$
V_{S}=V_{s b}+R V_{s b} \frac{\partial C}{\partial x} X_{2} \omega_{d r i v e} \sin \left(\omega_{\text {drive }} t\right),
$$

where $R$ is the trans-resistance amplifier gain. The gain $R$ can be adjusted in conjunction with the reference voltage $V_{R}$.

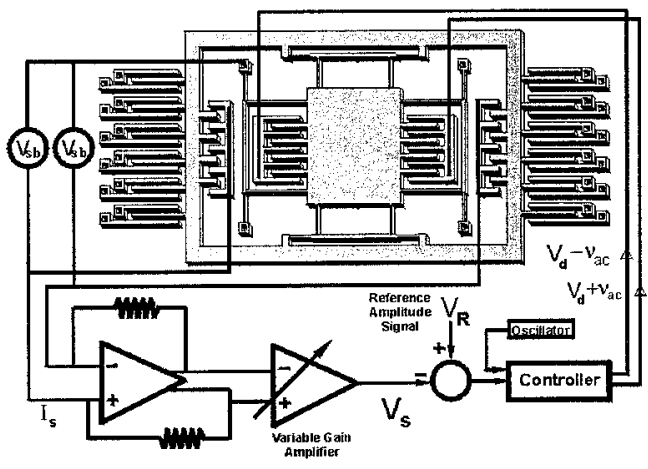

Figure 10: Closed-loop driving scheme of dual-mass gyroscope.

Once the error signal $e=V_{R}-V_{s}$ is obtained, it is fed into the controller. By summing the output voltage of the controller $V_{\text {cont }}$ and the nominal drive voltage $V_{\text {nom }}$, the controlled total drive voltage will be obtained to be fed to the comb-drives. A proportionalintegral controller with frequency modulator will drive the first mass with the required voltage amplitude and frequency to converge the error signal amplitude to zero. The controller should get the clock signal from an oscillator which will set the driving frequency $\omega_{\text {drive }}$ to the operational frequency.

When a system with $20 \%$ perturbation in the spring constants is simulated incorporating the proposed feedback controller, only $0.05 \mu m$ deviation is observed from the nominal drive oscillation amplitude of $1 \mu \mathrm{m}$. The same perturbation in the open-loop controlled system results in $0.28 \mu \mathrm{m}$ deviation (Fig. 11).

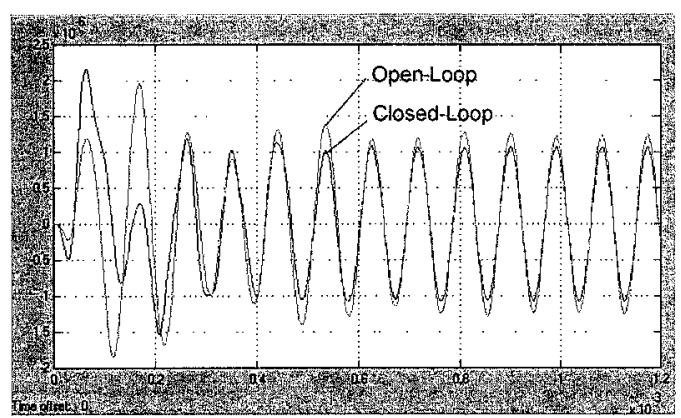

Figure 11: Closed-loop driving scheme of dual-mass gyroscope.

4.2 Compensation of Fabrication Imperfections An ideal gyroscope should be sensitive only to the input angular rate. However, in practice, micromachined vibratory gyroscopes are sensitive not only to the measured angular rate, but also to fabrication imperfections. Due to lack of perfect alignment of the intended and the actual principle axes of oscillation, anisoelasticity in the gyroscope structure occurs, causing dynamic cross-coupling between the drive and sense directions. The resulting dynamic cross-coupling stiffness and damping terms are the major factors that limit the performance of the gyroscope. Thus, the control system should eliminate the effects of the fabrication imperfections, and render the gyroscope sensitive only to the input angular rate.

\section{Noise Analysis in Sensing}

Micromachined Dual-Mass Gyroscope is basically a mechanical transducer which converts the input rotation rate to sense-direction oscillation. The deflection of the second mass due to the Coriolis force is converted into capacitance change by the sense capacitors. This capacitive signal should be converted into an output voltage signal with enough amplitude. The basic task of the sense electronics is to provide this conversion.

When the second mass deflects in sense direction, the finger attached to the mass (Figure 12) approaches finger A decreasing the capacitance $C_{s+}$, and moves away from finger $\mathrm{B}$ increasing the capacitance $C_{s-}$. The movement of the finger resulting from the deflection will translate the displacement to a change in capacitance. The purpose of the sense electronics is to detect this small capacitance change resulting from the very small displacements of the second mass, which are

$$
C_{s+}=C_{s-}=N \frac{\varepsilon_{0} t l}{y_{0}} .
$$

where $N$ is he total number of sense capacitors, $l$ is the length and $t$ is the thickness of the fingers $A$ and $\mathrm{B}$, and $y_{0}$ is the finger separation. 

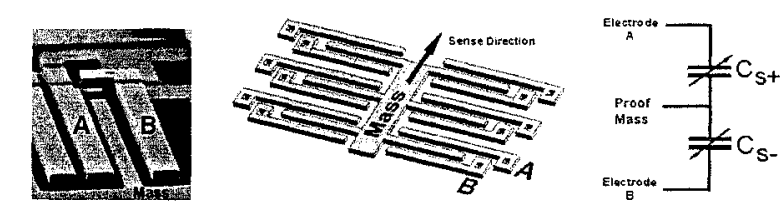

Figure 12: The actual differential sense capacitor fingers, and the circuit model of the capacitors.

\subsection{Parasitic Capacitances}

In the ideal case, the electrodes $\mathrm{A}$ and $\mathrm{B}$ in the comb structure interact with only proof-mass as parallel plates. However, the undesirable interaction in between the electrodes and the substrate introduces parasitic capacitances. The lumped-parameter model of the lateral sense-element consisting of sense capacitors $\left(C_{s+}\right.$ and $\left.C_{s-}\right)$, parasitic capacitors (electrode A - electrode B electrode capacitance $C_{a b}$, electrode A substrate capacitance $C_{a s}$, electrode B - substrate capacitance $C_{b s}$, and proof mass - substrate capacitance $\left.C_{p m s}\right)$, and parasitic resistors $\left(R_{a}\right.$ and $R_{b}$, and $\left.R_{p m}\right)$ is presented in Figure 13.

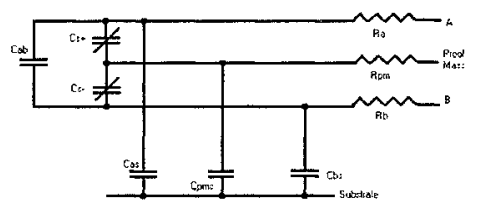

Figure 13: Electrical interface of sense electronics with parasitic capacitances.

\subsection{Integrator Implementation}

Small changes in capacitance due to Coriolis response result in a displacement current that flows through the sense capacitors. This current can be sensed employing an integrator as a sense amplifier configuration [2]. The integrator collects the charge on the integrator capacitance $C_{\text {int }}=2 C_{s}$ and outputs a low impedance voltage (Figure 14) with a transfer function of

$$
\nu_{\text {out }}=\frac{2 y}{C_{\text {int }}} \frac{\partial C_{s}}{\partial y} V_{s} \text {. }
$$

The integrator circuit introduces electrical noise generated by the op-amp in sensing. The effect of this output noise on measured input rotation rate should be analyzed for estimation of minimum detectable input rotation rate of the gyroscope. Given an input noise ratio $\overline{\nu_{n}^{2}}$, the overall noise $\overline{\nu_{o}}$ at the output of the integrator is [6]

$$
\overline{\nu_{o}^{2}}=\frac{C_{T}}{C_{i n t}} \overline{\nu^{2}{ }_{n}}=\frac{C_{X}}{C_{i n t}}\left(\nu_{t h_{s u s p}}^{2}+\nu_{t h_{i n t}}^{2}+\nu_{a m p}^{2}\right) .
$$

The input noise ratio $\overline{\nu_{n}^{2}}$ consists of op-amp input noise ratio $\overline{\nu_{a m p}^{2}}$, and the thermal noise from the suspension resistance $\left(\nu_{t h_{s u s p}}^{2}=\sqrt{4 k_{B} T R_{s u s p} B W}\right)$, and interconnection resistance $\left(\nu_{t h_{i n t}}^{2}=\sqrt{4 k_{B} T R_{i n t} B W}\right)$. Due to the increase in minimum detectable signal because of the thermal noise of the resistance introduced by the interconnections in off-chip sensing, improved performance is achieved by integrating sensing electronics on the same chip as the device. Thus, it is observed that noise in sensing electronics contribute to the gyroscope performance limitations as much as perturbations to be compensated by control electronics.

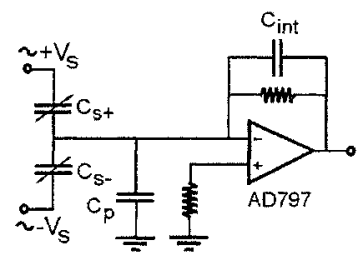

Figure 14: The implementation of the integrator for capacitive sensing [6].

\section{Conclusion}

In this paper, a novel micromachined vibratory rate gyroscope design approach which comprises of a 4-DOF dynamical system is presented. The control issues of the MEMS implementation of the design concept are addressed, and a control strategy for constant amplitude oscillation in the drive mode was developed. The proposed design is demonstrated to provide inherent robustness against fabrication variations, and fluctuations in the ambient temperature or pressure during the operation time of the device; requiring less demanding control strategies for operation under presence of perturbations. With the new design concept, passive disturbance-rejection is achieved by the inertial oscillator system, and it is verified that the complexity of the control electronics can be shifted to dynamical system complexity.

\section{References}

[1] N. Yazdi, F. Ayazi, and K. Najafi. Micromachined Inertial Sensors. Proc. of IEEE, Vol. 86, No. 8, August 1998.

[2] W.A. Clark. Micromachined Vibratory Rate Gyroscope. Ph.D. Thesis, BSAC, U.C. Berkeley, 1994.

[3] E. Netzer, and I. Porat. A Novel Vibratory Device for Angular Rate Measurement. Journal of Dynamic Systems, Measurement and Control, Dec. 1995.

[4] A. Shkel, R. Horowitz, A. Seshia, S. Park and R.T. Howe. Dynamics and Control of Micromachined Gyroscopes American Control Conference, CA ,1999.

[5] C. Acar and A. Shkel. Wide Bandwidth Micromachined Gyroscope to Measure Rotation. Patent pending, UCI Office of Technology Alliances, Case No:2001-140-1.

[6] A. Seshia. Design and Modeling of a Dual Mass SOI-MEMS Gyroscope. M.S. Thesis, BSAC, U.C. Berkeley, 1999. 